\title{
Tap Testing of Composites Benchmarked with Digital Shearography
}

\author{
Jasson Gryzagoridis ${ }^{1}$, Dirk Findeis ${ }^{2}$ \\ Mechanical Engineering Department \\ ${ }^{1,2}$ University of Cape Town \\ Cape Town - South Africa \\ E- Mail: jasson.gryzagoridis@uct.ac.za
}

\begin{abstract}
This paper reports on two non-destructive testing techniques employed to detect subsurface defects in composite materials of both the monolithic and sandwich types. Making use of the NDT techniques named on the title of this paper, Digital Shearography was used to benchmark the results obtained through the Tap Testing method. The tap testing was performed using the Mitsui Woodpecker WP-632AM, a hand held automated impact instrument that displayed the defects' positional data (X-Y coordinates) on a PC in real time. The versatility and ease of use of the Woodpecker is abundantly demonstrated and the comparisons with the results obtained with Digital Shearography on the same test specimens have given an indication of its accuracy.
\end{abstract}

Keywords: Coin/Tap testing, Digital Shearography, Nondestructive testing, Composite materials, Laminates, Sandwich Composites, Voids, Delaminations, Benchmarking.

\subsection{Introduction}

In the past few decades, we have been witnessing drastic changes in materials' technology. In the quest for improved strength to weight ratio composite materials appear to be the solution, however the advent of 'composites' has also brought about certain challenges. For example during the manufacturing stage and of course in their subsequent life during operation, flaws and defects will eventually appear in engineering components made from these materials. Traditional nondestructive testing (NDT) techniques have been attempting to a large extent keep up with the demands posed by the new materials, however it has become apparent that emerging and advanced NDT techniques are needed to bridge the widening gap of detecting and quantifying these defects, thus informing on action to avoid catastrophic events.

Some of the traditional NDT methods have been enhanced with modern state of the art gadgetry making them competitive with the newly advanced and emerging NDT techniques. This paper aims at benchmarking a traditional NDT technique which has been enhanced with modern tools (Tap Testing) by employing an emerging optical laser based NDT method (Digital Shearography), recognized for its high sensitivity in detecting flaws in composite materials.

\subsection{Tap Testing}

Tap Testing AKA Coin Testing is a very old, extremely simple NDT technique that involves literally the knocking on the surface of a component with a small hammer or a coin (hence the second name). This local excitation of the component is in fact a vibration test and one of the responses is the sound that emanates from the test piece. The physics of the coin-tap method have been very well investigated and explained by [1]. If a component 
contains a defect such as for example a disbond or delamination, a duller sound will be heard which will be the result of how the presence of the defect influenced the damping of the impact. The fact that an inspector has to listen to the sound that is produced when the surface of the test piece is knocked, suggests questionable reliability in identifying the presence of a defect and that the method is highly inspector-dependent.

The Tap Testing method when applied manually does not require or offer much of input and output data therefore attempts have been made to increase the reliability of the method by recording input and output parameters. In the case of automated mechanical impact testing the magnitude of the tap force or the duration of the impact are recorded through a sensor normally mounted behind the hammer used to produce the impact.

There is evidence of the development of automated systems recording the input and output data and displaying a visual image of the local surface stiffness, affording the possibility of not only detecting the presence of a flaw but also determining its size and location as well as a permanent record of the test.

The Rapid Damage Detection Device (WichitecRD3) from Wichitech Industries, the Computer-Aided Tap Testing (CATT) from Advanced Structural Imaging Inc. [2] and the Woodpecker from Mitsui/JR Technologies are examples of the level of sophistication that Tap Testing has attained.

The latest version of the Mitsui Woodpecker (WP-632AM) incorporates a numerical display into the facia of the hand-piece and can be purchased with an X/Y plotter which when used in conjunction with a pc or laptop is possible to display a colour tile map of the area under test. The instrument with its built-in sensors measures the contact time between the solenoid hammer and the test piece surface. A composite component that may contain defects such as damaged core, delamination, disbond, etc. will exhibit a longer contact time over the defect because the damage area offers less support than its undamaged part.

The contact time or duration of the impact is dependent on the stiffness underneath the surface and can be expressed by the following equation explained in [3]:

$$
\tau=\pi(\mathrm{m} / \mathrm{k})^{1 / 2}
$$

Where $\tau$ is the contact time or impact time, $\mathrm{m}$ is the mass of the instrument as it rests on the surface and $\mathrm{k}$ is the local stiffness of the material.

\subsection{Digital Shearography}

Shearography has evolved notably to this date, in its digital format, since its introduction by Hung [4]. Slowly but surely the method has become one of the major preferred techniques for nondestructive testing/evaluation for defects or flaws in structures made of composite materials. "The technique is a laser based optical method that determines the gradients of surface displacements on a test piece. It is an interferometric technique that relies on combining two speckled images of the surface of the component under inspection; one before and the other one after the specimen has been perturbed mildly for example by applying heat, pressure or vacuum, or a mechanical force"[5].

Much has been written in the relevant literature regarding the mathematical background of Shearography as well as descriptions of laboratory and portable industrial Shearography systems $[4,6,7$.$] . It will suffice here to reiterate that Shearography as a NDT$ technique offers advantages such as; being non-contact and non-invasive, provides whole field view, observations are made essentially in real time and is capable of normal to the surface strain detection sensitivity in any direction, of the order of half the wavelength of laser utilized, as can be deduced from equation 2: 


$$
\frac{\partial \delta p}{\partial n}=\frac{\lambda N}{2 S}
$$

where the gradient of the surface's normal displacement in any direction $(n)$ is proportional to product of the laser wavelength $(\lambda)$ and the number of fringes $(N)$ and inversely proportional to the image shear distance $(S)$.

Shearography featured as one of the devices/techniques employed in an inspection reliability study of advanced NDT methods conducted by FAA. In an example of results obtained from the probability of detection (POD) of various sizes of defect, Shearography performed very well compared to all other techniques, with no false calls and high POD for minimum flaw sizes [8].

\subsection{Experimental procedure}

\subsection{Shearography Testing}

The tests, on samples of composites that contained induced defects, were performed using the authors' Digital Shearography setup (Fig. 1) on the optical bench in their NDT laboratory at the University of Cape Town. The aim was to establish whether the defects in the test specimens were detectable by Shearography in which case, supported also by the FAA findings [8], a benchmark would be established for the Tap Testing results that would follow.

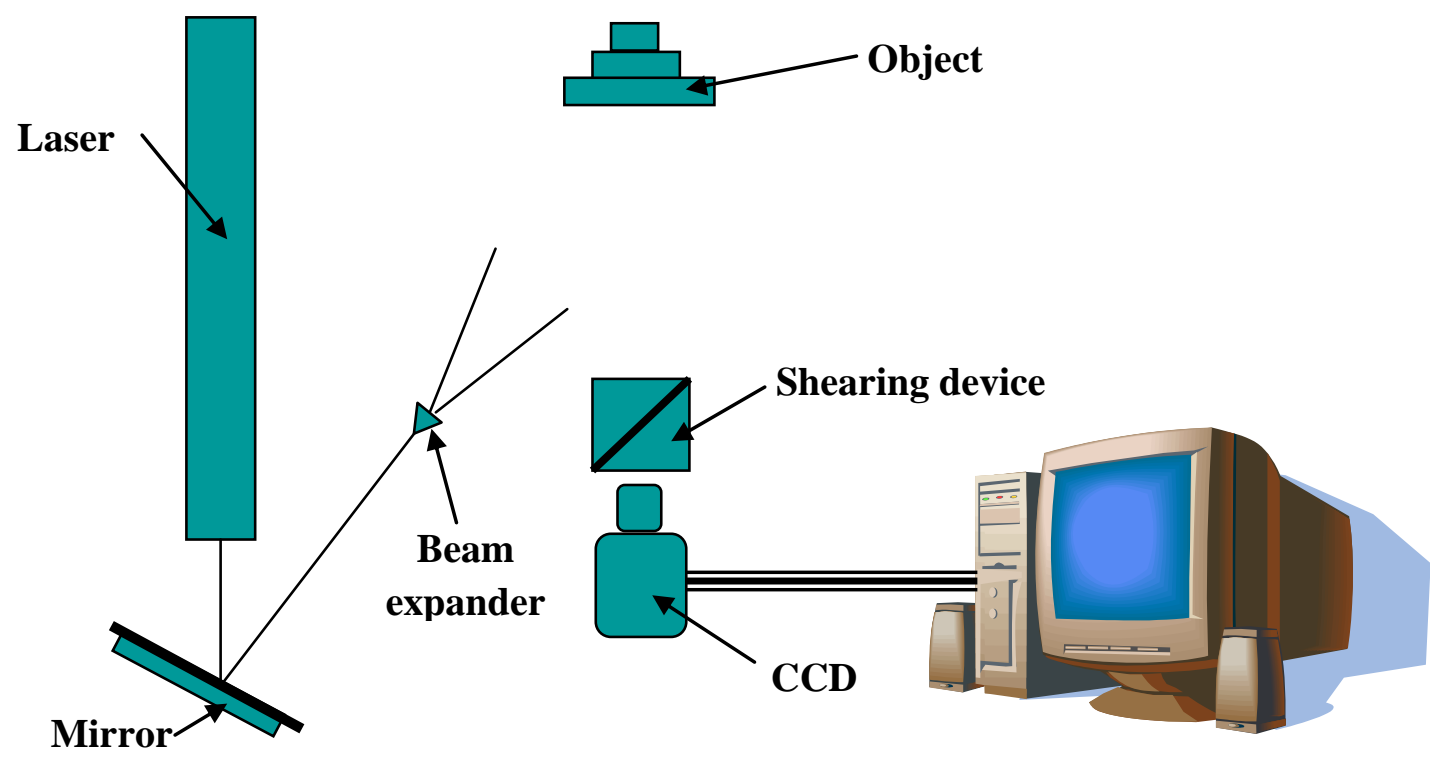

Figure 1: Schematic diagram of Digital Shearography set-up

The testing procedure simply consisted of exposing the test specimens to the laser illumination and with the PC based Digital Shearography running, a perturbation was induced to the specimens by warming up their surface with hot air from a hair drier for a few seconds. While observing on the monitor the dynamic fringe pattern on the shearographic images depicting the presence of the flaw beneath the test surface (as the specimen cooled to ambient conditions) a decision was made when to freeze/store the pattern in the computer. 


\subsection{Woodpecker Tap testing}

Following the testing of the composite specimens using the Digital Shearography technique described in 2.1 above, the authors proceeded in testing the same specimens using the Mitsui Woodpecker (WP-632AM). The instrument as depicted in fig. 2, in addition to displaying numerically the contact time of the hammer on the surface also is equipped with an $\mathrm{X} / \mathrm{Y}$ plotter linked to a $\mathrm{pc}$, which enabled simultaneous observation of the result as a colour coded tile. Scanning the test piece's surface in addition to storing the numerical results, which are referenced to the contact time of a defect free specimen, also produces a map of these tiles with colours that might range through green, yellow, brown, purple and red depending on the magnitude of disagreement of the 'local' contact time to the (green) reference one. Therefore observing the map of the coloured tiles (other than green) one gets a feel of the extent/size of the defect and depending on the colour one gets a feel of the degree of disparity with the value of a defect free result.
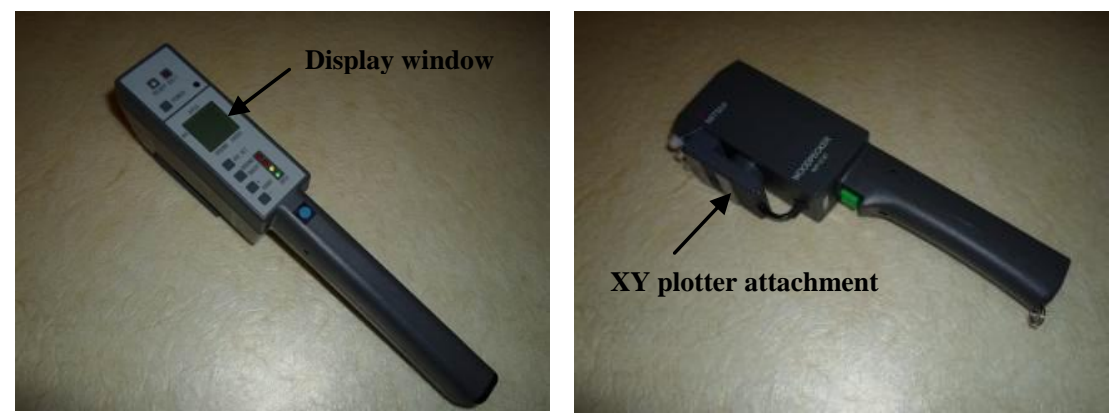

Figure 2. Images of the Mitsui Woodpecker (WP-632AM) showing the display face (left) and $\mathrm{X} / \mathrm{Y}$ plotter attachment at the feet of the instrument (right)

\subsection{The test specimens}

Four test specimens with manufactured defects were prepared for the Shearography and Tap Testing procedures. The specimens are depicted in fig. 3 and in addition their particular details regarding their construction, materials used to make them, their overall dimensions and type of defect were as follows:

Test specimen \# 1 was a sandwich composite beam made with $1.5 \mathrm{~mm}$ fibreglass skins and $9.0 \mathrm{~mm}$ balsa core. Overall dimensions were: length $310.0 \mathrm{~mm}$, width $40.0 \mathrm{~mm}$, and thickness $12.0 \mathrm{~mm}$. The defect was manufactured near the middle of the beam by inserting a blade between the skin and the core at the edge of the beam thus creating a void $30.0 \mathrm{~mm}$ long and $20 \mathrm{~mm}$ deep.

Test specimen \# 2 was made by bonding together two Perspex sheets of thickness 2.0 and $3.0 \mathrm{~mm}$ respectively having the overall dimensions of $210.0 \mathrm{~mm}$ squares. In the middle of the specimen, two thin Teflon sheets $25.0 \mathrm{~mm}$ and $15.0 \mathrm{~mm}$ squares were placed between the two sheets, creating respective inserts with centres $70.0 \mathrm{~mm}$ apart.

Test specimen \# 3 was manufactured using $9.0 \mathrm{~mm}$ thick foam core sandwiched between $3.0 \mathrm{~mm}$ thick fibreglass skins. The overall dimensions of the specimen were length $450.0 \mathrm{~mm}$, width $150.0 \mathrm{~mm}$ and thickness $15.0 \mathrm{~mm}$. For this specimen four flat bottom holes of $20.0 \mathrm{~mm}$ diameter were prepared at a depth of 12.0, 7.0, 5.0, and $3.0 \mathrm{~mm}$ from the surface of the skin.

Test specimen \# 4 was a replica in construction, materials used, and having overall dimensions identical to those of specimen \#3. It differed on the type of defects that were manufactured as flat bottom holes of 25.0, 20.0, 15.0, and $10.0 \mathrm{~mm}$ diameter at a depth of 5.0 $\mathrm{mm}$ from the surface of the skin. 


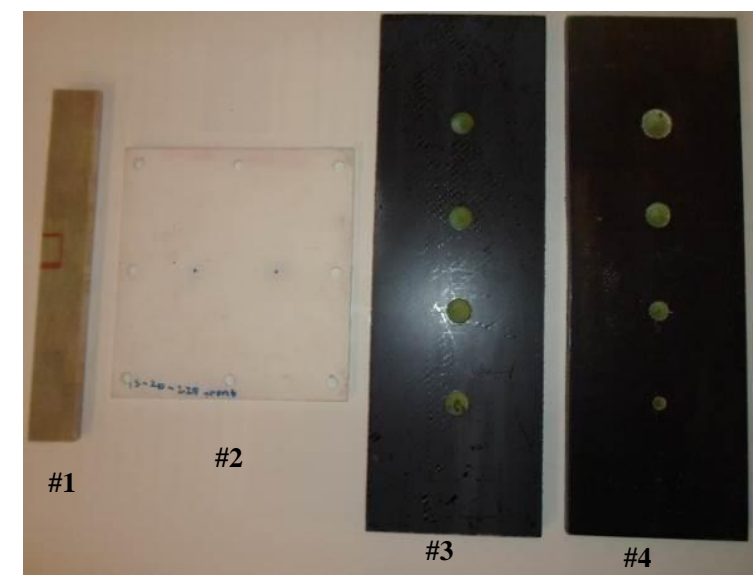

Figure 3. Images of the test specimens used in the experiments

\subsection{Results and Comments}

The experimental results, using the Digital Shearography and the Tap Testing techniques, when attempting to detect the manufactured defects on the test specimens as described in the text and depicted in fig. 3 above, are presented side by side in order to enable direct comparison and to facilitate the interpretation.

\section{Results from specimen \#1}

The collage of images presented in fig. 4 serves to demonstrate that the induced defect (a void between skin and core) was identified easily with both techniques regardless if the inspection took place on the near or far side surface of the specimen, relative to the location of the defect. As expected the detect ability of both techniques suffered mildly when attempting to test with the defect located in the far side of the specimen.
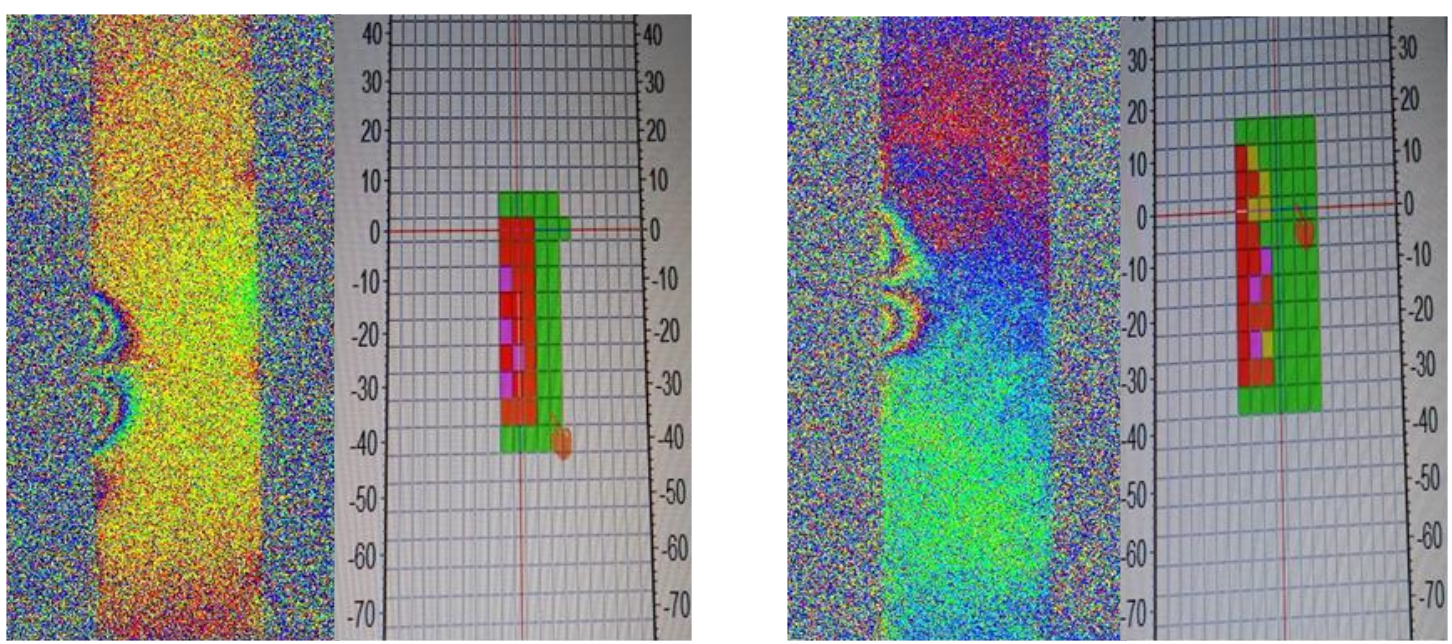

Figure 4 Results from specimen \#1 - (left) Near surface Shearography and Tap Testing results, (right) far side Shearography and Tap Testing results respectively 


\section{Results from specimen \#2}

The results of the test performed on specimen \#2 equally demonstrate that both techniques were capable in detecting, in this case, as is depicted in figure 5 below, the two Teflon inserts bonded between the two Perspex sheets. No attempt was made to differentiate between near and far surface testing, in that the difference in thickness of the material from surface to the defect was negligible.
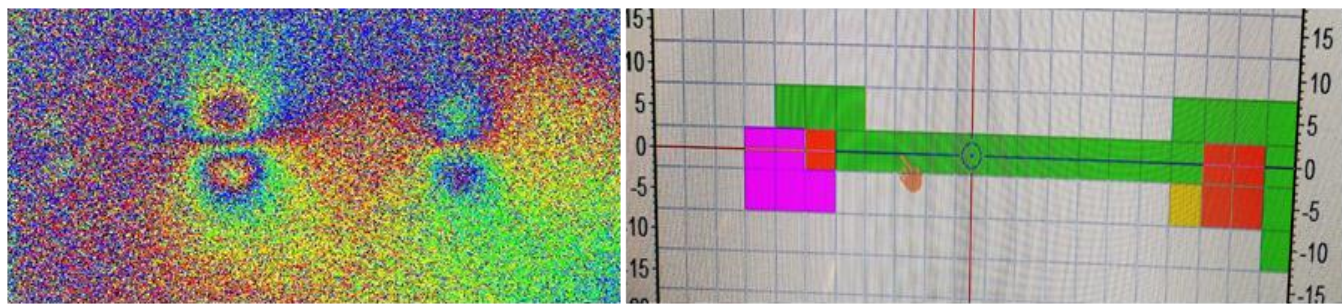

Figure 5. Shearography and Tap Testing results left and right respectively, of the detection of the Teflon inserts between two Perspex sheets

\section{Results from specimen \#3}

Specimen \#3 presented more challenging conditions to the Shearography and Tap Testing techniques in that the material of the core of the composite was foam which is not only a poor conductor of heat but also a fairly reasonable vibrations damping agent. However, both techniques demonstrated their ability to detect a $20 \mathrm{~mm}$ dia. blind hole at all depths from the testing surface.
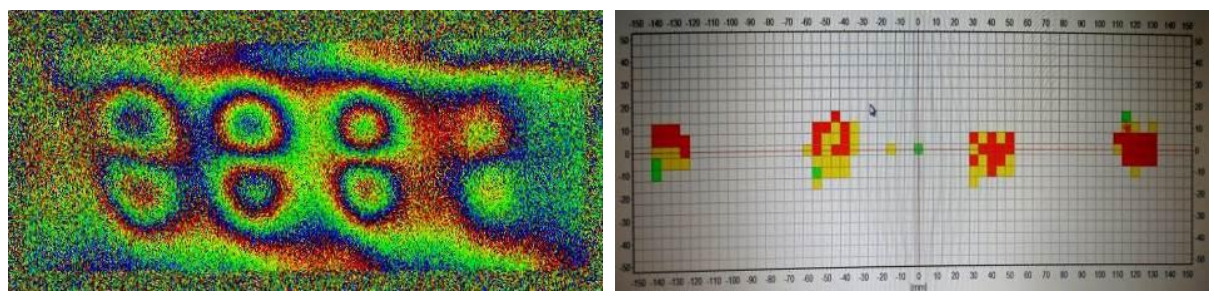

Figure 6. Flat bottom holes at different depths from the test surface adequately detected by Shearography on the left and Tap Testing on the right

\section{Results from specimen \#4}

The final tests reported here were conducted on the specimen that had the four flat bottom holes of varying diameters all at the same depth from the test surface.
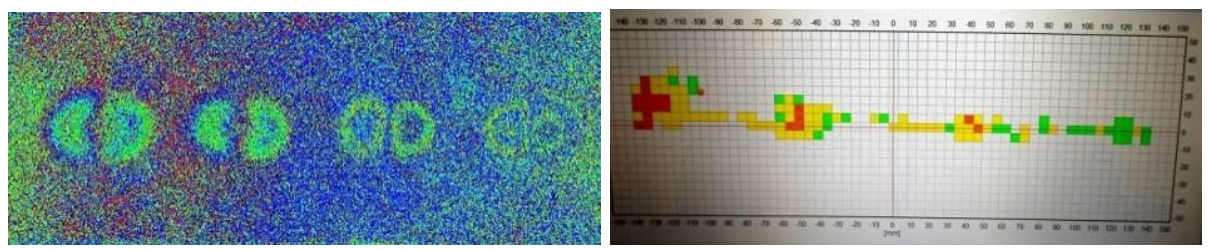

Figure 7. Different dia. flat bottom holes at a depth of $5.0 \mathrm{~mm}$ from the surface as recorded by the Shearography and Tap Testing tests respectively. 
It was demonstrated again that Digital Shearography is capable of detecting various manufactured defects; however the Tap Testing method appears to have reached the limit of its ability to detect a defect somewhere between the size of the 15.0 and $10.0 \mathrm{~mm}$ dia. flat bottom holes, for the particular type of core material used in the manufacture of this composite specimen. This is seen on the colour tile map or record of the test (see the image on the right in fig. 7), where there are only a couple of red coloured tiles for the $15.0 \mathrm{~mm}$ dia. hole (third from the left). The $10 \mathrm{~mm}$ dia. hole located at the extreme right of the specimen produced no colour tiles. What is also noticeable in this figure is a perception of the diminishing size of the defects from left to right in the Shearography image, as well as the amount of red colour tiles associated with each defect on the Tap Testing tile colour map.

Finally we can conclude that compared to Digital Shearography the Tap Testing technique is fairly capable of detecting defects in monolithic and sandwich type composites. The particular instrument employed to perform the Tap Testing NDT technique known as the Woodpecker was judged to be user friendly, requiring minimum preparation or set-up time yielding readily a record of the test results in pictorial and numerical format. Further work is envisaged in establishing the technique's/instrument's ability to establish the minimum size of defect that it can detect and if this is influenced by the particular composite material involved in the construction of a component.

\section{Acknowledgements}

The authors wish to thank Armscor (Armaments Corporation of South Africa Ltd.) for their continuous support in the research work on aspects of Non Destructive Evaluation being carried out at the University of Cape Town, South Africa.

\section{References}

1. Cawley P and Adams R.D: "The Mechanics of the Coin-Tap Method of NonDestructive testing", Journal of Sound and Vibration, 1988, 122(2), 299 316

2. www.asi-nde.com/usingthecatt/using the catt.html: Using the CATT - Advanced Structural Imaging. Last accessed during April 2013.

3. Cawley P: "High Frequency Coin-Tap Method Of Non-Destructive Testing" Mechanical Systems and Signal Processing, (1991), 5(1), 1 11.

4. Hung YY: Shearography: a new optical method for strain measurement and nondestructive testing: Opt. Eng. 21 No.3, 391-395 (1982)

5. Gryzagoridis J, Findeis D, Musonda V "Using Digital Shearography to Visualize and Quantify Defects in Composite Aerospace Components".CD -Proceedings of the $8^{\text {th }}$ South African Conference on Computational and Applied Mechanics-SACAM08 Cape Town, 26-28 March 2008

6. Gryzagoridis J, "Laser Based Nondestructive Inspection Techniques” J Nondestruct. Eval. 31 (4), pp. 295-302 (2012)

7. Guowen, L., Benjamin, A., Bard, B.A., Shudong, W.: Real-time portable phasestepping shearography system for NDE. Proc.SPIE 3397, 156-168 (1998)

8. Galela D: "FAA Inspection Research Activities for Composite Materials" The 2006 Composite Damage Tolerance \& Maintenance Workshop, ATO-P, July 20, (2006) 